\title{
Disease-Specific Induced Pluripotent Stem Cell Modeling: Insights into the Pathophysiology of Valosin Containing Protein (VCP) Disease
}

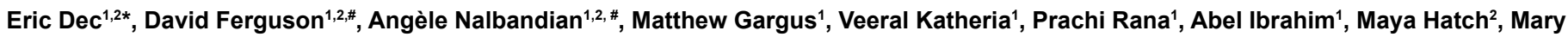
Lan $^{1}$, Katrina J Llewellyn ${ }^{1}$, Hans Keirstead ${ }^{2}$ and Virginia E Kimonis ${ }^{1,2 *}$

${ }^{1}$ Department of Pediatrics, Division of Genetics and Metabolism, University of California-Irvine, Irvine, CA 92697, USA

${ }^{2}$ Anatomy and Neurobiology, Reese-Irvine Research Center, Sue and Bill Gross Stem Cell Research Center, University of California-Irvine, Irvine, CA 92697, USA

\#The authors wish it to be known that, in their opinion, the first three authors should be regarded as joint First Authors.

\begin{abstract}
Valosin Containing Protein (VCP) disease is an autosomal dominant disorder caused by mutations in the VCP gene and is associated with progressive muscle weakness and atrophy. Affected individuals exhibit striking scapular winging due to shoulder girdle weakness. Currently, there are no treatments available and patients are dying early from cardiac and respiratory failure, typically in their 40's and 50's. The generation of disease-specific induced pluripotent stem cells (iPSC) offers a novel platform to investigate mechanisms of VCP disease and potential treatments similar to other disease models including Amyotrophic Lateral Sclerosis (ALS), Duchenne muscular dystrophy (DMD), Parkinson's disease (PD), Alzheimer's disease (AD), Best Disease (BD), and type I juvenile diabetes mellitus (T1DM). Herein, we report the generation and characterization of a human iPSC line to examine the cellular and molecular processes underlying VCP disease. The VCP iPSC line expressed specific pluripotency markers NANOG, SSEA4, OCT-4, TRA-1-81 and exhibited characteristic morphology. We differentiated the human iPSC cell line into a neuronal lineage confirmed by TUJ-1 staining, a neuronal class III $\beta$-tubulin marker. We detected higher protein expression levels of ubiquitin (Ub), TAR DNA-binding protein-43 (TDP-43), Light Chain 3-I/II (LC3), p62/SQSTM1, and optineurin (OPN) in the iPSC neural lineage compared to the control neural line. Collectively, our results demonstrate that patient-specific iPSC technology may provide useful disease modeling for understanding the complex mechanisms and for developing novel treatments of VCP and related disorders.
\end{abstract}

Keywords: iPS cell VCP disease modeling; Multisystem proteinopathy; Hereditary inclusion body myopathy; Paget disease of bone; Frontotemporal dementia; Valosin containing protein; autophagy

\section{Introduction}

Hereditary Inclusion Body Myopathy, Paget Disease of Bone, Frontotemporal Dementia (IBMPFD) and Amyotrophic Lateral Sclerosis (ALS), recently termed Multisystem Proteinopathy (MSP) is a multisystem disorder with an array of manifestations caused by mutations in the valosin containing protein $(V C P)$ gene $[1,2]$. Over 31 VCP mutations have been identified worldwide in families from several parts of the world including Germany [3,4], France [5], Austria [6], Italy [7,8], the United Kingdom [9], Australia [10], Brazil [11], Korea [12], Japan [13] and the United States [14,15], with the R155H mutation accounting for more than $50 \%$ of affected individuals. Varied additional manifestation of VCP mutations include ALS in $10-15 \%$ of patients, isolated familial ALS (fALS) which account for 2-3\% of fALS [2], Parkinson's disease (PD) [16], Alzheimer's disease (AD) [17], cardiomyopathy [18], sphincter disturbance [9], among other manifestations. Although VCP proteinopathy is a relatively rare disorder, exploration of its cellular and molecular mechanisms holds promise for explaining shared pathologies of more common proteinopathies such as ALS, PD, and FTD. VCP, a member of the type II AAA+ ATPase family, plays an important role in a plethora of cellular activities and recent studies have implicated the ubiquitin proteasome protein degradation pathway [19] and the autophagy pathway [20,21], mitochondrial quality control [22] and potentially other signaling pathways [23] in the pathogenesis of VCP-associated MSPs.

There are currently no known effective disease-modifying treatment options for the clinical manifestations of VCP-related neurodegenerative disease, except for Paget disease of bone (PDB) which can be treated effectively with bisphosphonates. Patients become wheel-chair bound and die prematurely from respiratory failure secondary to profound, progressive muscle weakness and cardiomyopathy. Histologically, patients display rimmed vacuoles and TDP-43-positive ubiquitinated inclusion bodies in muscle [18,24-26] and electron micrographs from affected skeletal muscle demonstrate prominent $15-21 \mathrm{nM}$ tubulofilamentous inclusions within myonuclei. PDB in addition to being present in 50\% of patients with VCP disease is also a common disease in the general population. PDB caused by overactive osteoclasts is associated with a disorganized bone turnover and can affect one or several bones throughout the axial and appendicular skeleton. PDB may result in bone deformity, pain, pathological fracture, deafness, and other nerve compression related problems. Onset of frontotemporal dementia (FTD), the third most common component of VCP disease, occurs on average at 54 years with an overall frequency of $33 \%$ [24]. Hallmarks of the brain pathology include inclusions of ubiquitin and TDP-43, also seen in other proteinopathies associated with FTD [27-32].

A recurrent, well-validated impact of VCP protein dysfunction

*Corresponding author: Virginia Kimonis, MD, MRCP, Department of Pediatrics, Division of Genetics and Metabolism, University of California-Irvine, 2501 Hewitt Hall, Irvine, CA 92697, USA, Tel: (714) 456-5791, (714) 456-2942; Fax: (714) 4565330; Pager: (714) 506-2063; E-mail: vkimonis@uci.edu

Received January 05, 2014; Accepted February 09, 2014; Published February 11, 2014

Citation: Dec E, Ferguson D, Nalbandian A, Gargus M, Katheria V, et al. (2014) Disease-Specific Induced Pluripotent Stem Cell Modeling: Insights into the Pathophysiology of Valosin Containing Protein (VCP) Disease. J Stem Cell Res Ther 4: 168. doi:10.4172/2157-7633.1000168

Copyright: (c) 2014 Dec E, et al. This is an open-access article distributed under the terms of the Creative Commons Attribution License, which permits unrestricted use, distribution, and reproduction in any medium, provided the original author and source are credited. 
is impaired autophagy. VCP is important for the retro-translocation of misfolded endoplasmic reticulum proteins, and failure in this activity results in defective endoplasmic reticulum-associated degradation (ERAD) as well as accumulation of ubiquitin aggregates and cytoplasmic translocation of TDP-43 [33-37]. Myoblasts from patients have revealed disruption of autophagy with autophagosome accumulation, and apoptosis as the pathogenetic mechanism for this disease [21]. Accumulation of autophagic markers such as p62/SQSTM1 and Light Chain 3-I/II (LC3) suggest upregulation of autophagy in the pathophysiology of VCP disease at the cellular level ultimately leading to myopathic degeneration. A recent study by Kim et al. [22] elucidated the role of VCP downstream of PINK1 in the PINK1/Parkin pathway and its recruitment to mitochondria in response to Parkin-mediated ubiquitination. Thus, VCP is not only important in autophagy, but also mitophagy, a process utilized in the clearance of damaged mitochondria [22].

A key challenge in the field has been to develop an in vitro disease model demonstrating the multiple disease-related phenotypes and pathogenesis of VCP disease. Renewable, accessible cell lines for the development of therapeutic drug trials and cell assays are critical. Here, we describe the generation and characterization of a VCP iPSC-derived cell line from an IBMPFD patient's fibroblasts and its differentiation into neurons modeling VCP disease. This report demonstrates the significant potential of using patient-specific iPSC technology to explore the pathophysiological mechanisms and to screen therapeutic drugs for VCP-associated neurodegenerative diseases.

\section{Materials and Methods}

\section{Reprogramming and maintenance of patient fibroblasts to iPSC}

Primary fibroblasts were obtained from a 57-year old female diagnosed with IBMPFD by both clinical signs and molecular sequence analysis. Fibroblasts were sent to UConn Stem Cell Core for reprogramming through the retroviral transfection system using Oct-4, Sox-2, KLF-4, c-MYC, Lin28 as described previously [38,39]. Briefly, human fibroblasts were maintained in Dulbecco's modified eagle medium (DMEM, Invitrogen, Carlsbad, CA) containing 10\% fetal bovine serum (FBS) and $0.5 \%$ penicillin and streptomycin. Human iPSC cells were plated onto mouse embryonic fibroblast feeders (Global Stem Inc., Rockville, MD) and maintained in knockout serum replacement- containing culture media supplemented with basic Fibroblast Growth Factor (bFGF) $(10 \mathrm{ng} / \mathrm{ml})$ per standard culture practices. For passaging, human iPS cells were washed with PBS and incubated with DMEM/F12/collagenase IV. Colonies were scraped off when confluent and seeded onto Matrigel-coated plates in MEFconditioned ES cell medium, supplemented with $4 \mathrm{ng} / \mathrm{ml}$ bFGF.

\section{Chromosome counting}

To analyze the karyotyping of the hiPSCs, cells were treated with Nocodazole and BrdU within 6 hours of feeding. Subsequently, cells were rinsed, trypsinized, washed and resuspended with $500 \mu \mathrm{l}$ of hESC media. Five ml of hypotonic phosphate buffer was slowly added. Samples were heated for 5 minutes at $37^{\circ} \mathrm{C}$ followed by the addition of 10 drops of fixative. Cell lysate was centrifuged and supernatant aspirated and again fixative was added. The solution was then dropped on to slides and allowed to dry over $70^{\circ} \mathrm{C}$ water bath. Slides were mounted with DAPI hard-set mounting medium (Vector Labs, Burlingame, CA) and chromosomes were counted.

\section{Sequence analysis}

Human iPSCs were cultured and DNA was extracted for sequence analysis (University of California-Irvine Mitomed Laboratory Services, Irvine, CA). Valosin-containing protein (VCP) sequence analysis was completed using the $\mathrm{ABI}$ sequencer with upstream and downstream VCP primers, which confirmed the presence of the VCP R155H mutation in the patient's native fibroblasts.

\section{Teratoma formation into three germ layer types}

A teratoma assay was performed at the Advanced Stem Cell Inc. (San Francisco, CA). Briefly, candidate iPSCs were injected into the kidney capsules of NOD/SCID mice. Six weeks post-injection, the kidney capsule mass was dissected, sectioned, and stained with standard hematoxylin and eosin (H\&E) for pathological purposes. Sections were analyzed by light microscopy.

\section{Differentiation of iPSCs into neurons}

Motor neuron (MN) differentiation was initiated by exposing the VCP pluripotent cell culture to a serum-free media DMEM: F12 supplemented with B-27 and Insulin-Selenite Transferrin (ITS) (Life Technologies, Carlsbad, CA). Cultures were dissociated the next day, transferred in ultra-low adherent flasks and supplemented with $10 \mathrm{ng} /$ $\mathrm{mL}$ bFGF and $10 \mu \mathrm{M}$ retinoic acid (RA) for the first 7 days, then only with $5 \mathrm{ng} / \mathrm{mL}$ bFGF for the duration of differentiation. The formation and growth of neurospheres were observed over the following 14 days, and cultures were then transferred to Matrigel-coated flasks (1:60) on day 21 . The adherent cultures were expanded for another 7-14 days in the same media and growth factors conditions. Motor neurons were harvested before reaching maturity by enzymatic dissociation and replated in low density on poly-d-lysine and laminin-coated surfaces in the absence of growth factors. Maturation was manifested about 7-14 days later by typical multi-branched neuronal morphology. Motor neurons derived from normal embryonic stem cells (California Stem Cell, Irvine, CA) were used for controls for ICC staining.

\section{Immunocytochemistry (ICC)}

iPSCs were seeded onto laminin-coated 4-well chamber slides and cultured in neuronal differentiation medium. At day 28 of differentiation, isolated patches of iPSCs were discernible by light microscopy depicting polygonal morphology and pigmentation. iPSCs were washed with PBS then fixed in $4 \%$ paraformaldehyde (PFA) for 15 minutes, and permeabilized with Triton X-100 for ICC staining. Primary antibody staining was carried out using OCT-4 (SigmaAldrich, St. Louis, MO), NANOG (Sigma-Aldrich), SSEA4 (GeneTex, Irvine, CA), and TRA-1-81 (Sigma-Aldrich) in a humidified chamber for 2 hours, then washed with 1X PBS. Sections were then incubated with secondary antibodies anti-rabbit conjugated FITC or TRITC (BD Biosciences, San Jose, CA) for 1 hour. Subsequently, slides were washed three times with 1X PBS and mounted with DAPI hard-set mounting medium. Sections were analyzed by light microscopy.

\section{Protein expression studies}

Patient iPSC (passage 9) and control neuronal cell line samples were cultured and proteins were extracted using the NE-PER Nuclear and Cytoplasmic Extraction Kit (Thermo Scientific, Rockford, IL). Protein concentrations were determined using the Nanodrop according to the manufacturer's protocols. Equal amount of proteins were separated on Bis-Tris 4-12\% NuPAGE gels using the Novex Mini Cell (Invitrogen Life Technologies, Carlsbad, CA) according to manufacturer's protocols. The expression levels of proteins: VCP, TUJ-1, Ubiquitin, 
Citation: Dec E, Ferguson D, Nalbandian A, Gargus M, Katheria V, et al. (2014) Disease-Specific Induced Pluripotent Stem Cell Modeling: Insights into the Pathophysiology of Valosin Containing Protein (VCP) Disease. J Stem Cell Res Ther 4: 168. doi:10.4172/2157-7633.1000168

Page 3 of 6

TDP-43, LC3-I/II, p62/SQSTM1, optineurin-specific antibodies were analyzed by Western blotting. Equal protein loading was confirmed by $\beta$-actin antibody (Santa Cruz Biotechnology, Santa Cruz, CA) staining. These experiments are representative of triplicates. Densitometry was performed to quantitate the Western blot bands using Image J Program (National Institutes of Health, Bethesda, MD).

\section{Results}

\section{Establishment and characterization of VCP disease patient iPSCs}

A fibroblast cell line was established from the skin biopsy obtained from a 57-year old female proband, the first patient described [18], harboring the most common VCP R155H mutation. This patient was diagnosed with classic clinical features including muscle weakness and Paget disease of bone affecting her cranium and hip. Fibroblasts were reprogrammed by lentiviral delivery of the following genes: OCT-4, SOX2, c-MYC, and KLF4 (UCONN HealthCare Core, Farmington, $\mathrm{CT})$. Sequencing analysis confirmed that the VCP patient iPSC line harbored the $\mathrm{R} 155 \mathrm{H}$ mutation [24]. The undifferentiated cells were co-stained with DAPI (Figures 1A and 1E) and pluripotent markers NANOG (Figure 1B), SSEA4 (Figure 1C), OCT4 (Figure 1F), and TRA-1-81 (Figure 1G) by immunocytochemistry (ICC) and analyzed by confocal microscopy. Merged overlay images of undifferentiated iPS cells with DAPI nuclear stain (Figures 1D and 1H). Karyotyping and sequencing analyses were assessed. Fifty metaphase spreads were counted all having 46 chromosomes (data not shown).

\section{Confirmation of iPSC generation by teratoma formation}

Teratoma assays were performed to check for the pluripotency of each iPSC line. NOD/SCID mice were injected with the newly generated human iPSC line and monitored for six weeks for teratoma formation to subsequently analyze gross morphology. By hematoxylin and eosin staining. Immunocytochemical analyses of the teratomas demonstrated distinct features all three germ layers including the endoderm, mesoderm, and ectoderm confirming pluripotency (Figure 2). Endodermal structures included glands (Figure 2A) and ducts (Figure 2B). Sections of the mesoderm revealed (Figure 2C) cartilage,
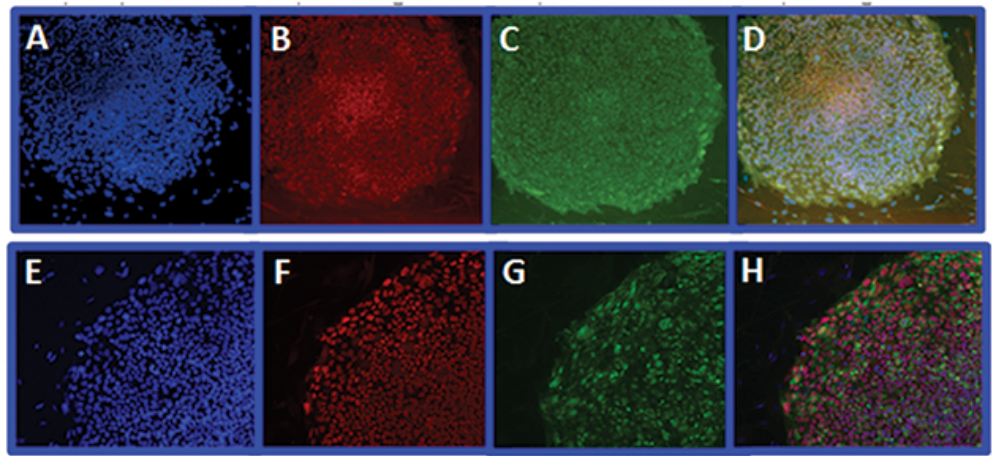

Figure 1: Generation and immunohistochemical characterization of patient and control iPSC lines. Human iPSC from a 57-year old patient diagnosed with IBMPFD were stained with (A and E) DAPI (nuclei), two internal nuclear markers (B) NANOG and (F) OCT-4 and two external surface markers (C) SSEA-4 and (G) TRA-1-81. (D and $\mathrm{H}$ ) Merged overlay images of stained iPSC with DAPI. (Magnification 200X).

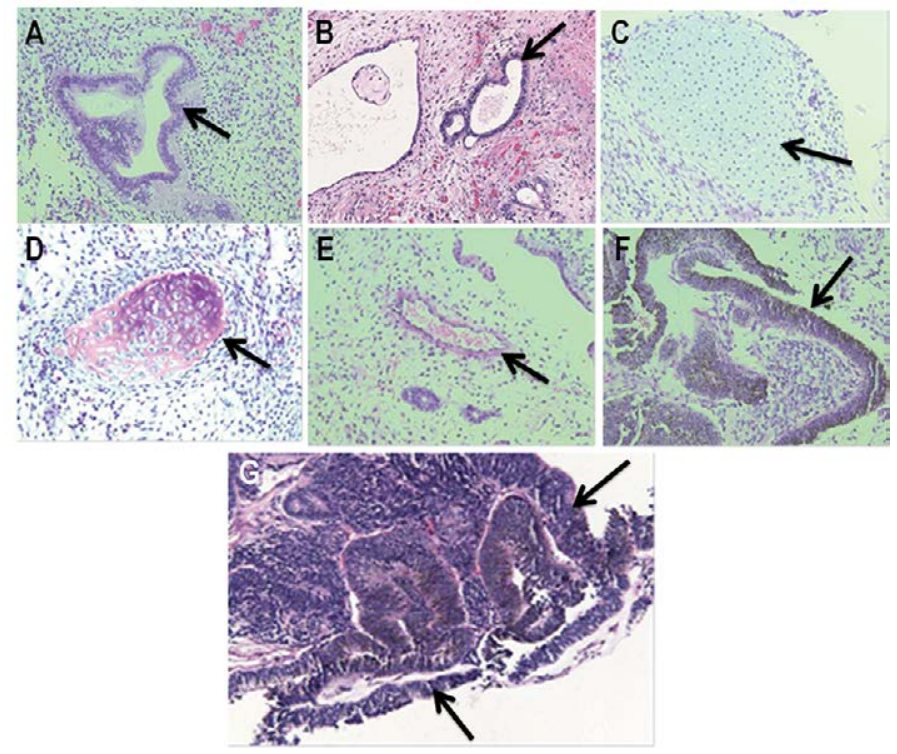

Figure 2: Teratoma formation from human iPSC in NOD/SCID mice. Teratoma staining in NOD/SCID mice demonstrated formation of all three germ layers. Endodermal structures included (A) glands and (B) ducts. Sections of the mesoderm revealed (C) cartilage, (D) ossifying cartilage, and (E) blood vessel formation. The ectoderm contained $(F)$ pigmented cells in addition to $(G)$ neuronal rosette formation (shown by black arrows). (Magnification $200 X)$. 
Citation: Dec E, Ferguson D, Nalbandian A, Gargus M, Katheria V, et al. (2014) Disease-Specific Induced Pluripotent Stem Cell Modeling: Insights into the Pathophysiology of Valosin Containing Protein (VCP) Disease. J Stem Cell Res Ther 4: 168. doi:10.4172/2157-7633.1000168

Page 4 of 6

ossifying cartilage (Figure 2D), and blood vessel formation (Figure 2E). The ectoderm contained pigmented cells (Figure $2 \mathrm{~F}$ ) in addition to neuronal rosette formation (shown by arrows) (Figure 2G).

\section{Differentiation of iPSCs into neuronal lineage}

Differentiation of VCP iPSC into neurons was performed according to our established protocols with a slight modification. Patient iPSCs and the unaffected control line colony formation were analyzed by light microscopy prior to differentiation (Figures $3 \mathrm{~A}$ and $3 \mathrm{~B}$ ) and post-differentiation into motor neurons at day 8 (Figure 3C). Day 42 VCP iPSC were double-stained with glial fibrillary acidic protein (GFAP) and neuron-specific class III beta-tubulin (TUJ-1) which validated differentiation into the neuronal lineage (Figure 3D). For every experiment, patient-specific iPSCs were cultured and passaged in parallel for the same period of time under the same conditions.

\section{Increased accumulation of autophagy signaling markers in differentiated iPSCs}

The autophagy cascade whereby long-lived proteins are degraded is of critical importance in understanding one of the possible underlying mechanisms in VCP disease. We and others have previously shown that the autophagic pathway is disrupted in patients, myoblasts and as well as in our $\mathrm{VCP}^{\mathrm{R} 155 \mathrm{H} /+}$ mouse model $[21,40,41]$. To determine the pathophysiological effects of $V C P$ mutations on the patient iPSC line, we analyzed the autophagy markers TDP-43 (Figures $4 \mathrm{~A}$ and 4B), LC3-I/II (Figures 4C and 4D), p62/SQSTM1 (Figures 4E and $4 \mathrm{~F}$ ) by immunohistochemistry and Western blotting (Figure $4 \mathrm{G}$ ). As compared to the control line, the patient iPSC line demonstrated increased protein expression levels of ubiquitin, TDP-43 and the autophagic markers: LC3-I/II, p62/SQSTM1, and optineurin (OPN) thereby suggesting impaired degradation of the proteins involved in the autophagosome-lysosomal cascade (Figure 4G). Levels of VCP and TUJ-1 remained comparable between the control and iPSC line (Figure $4 \mathrm{G}) . \beta$-actin was used as a loading control and densitometry confirmed these findings.

\section{Discussion}

Hereditary inclusion body myopathy (h-IBM) characterized by rimmed vacuoles and ubiquitin and TDP-43-positive large inclusion bodies in the muscles was first recognized by Kimonis et al. [18,2426]. VCP disease pathology encompasses multisystem pathologies including PDB and/or FTD, ALS [26,42,43], and cardiomyopathy among other disorders. Recently, studies have demonstrated motor neuron involvement in multisystem proteinopathies with potential relevance to the pathobiology of ALS [44]. ALS is an important and devastating component of VCP disease with VCP mutations (R155H, R159G, R155C) being identified in 6 families. Hence, we chose to differentiate our hiPSC into a neuronal cell lineage to examine the in vitro pathological changes including cellular and molecular mechanisms of autophagy.

To the best of our knowledge, this is the first study to recapitulate the VCP disease phenotype in an iPSC model system. These cellular models will allow us to evaluate the cellular effects of specific gene mutations as well as to analyze physiologically relevant treatments to better understand genotype-phenotype correlations in vitro. Although primary cell lines are the mainstay of in vitro research, viable patient
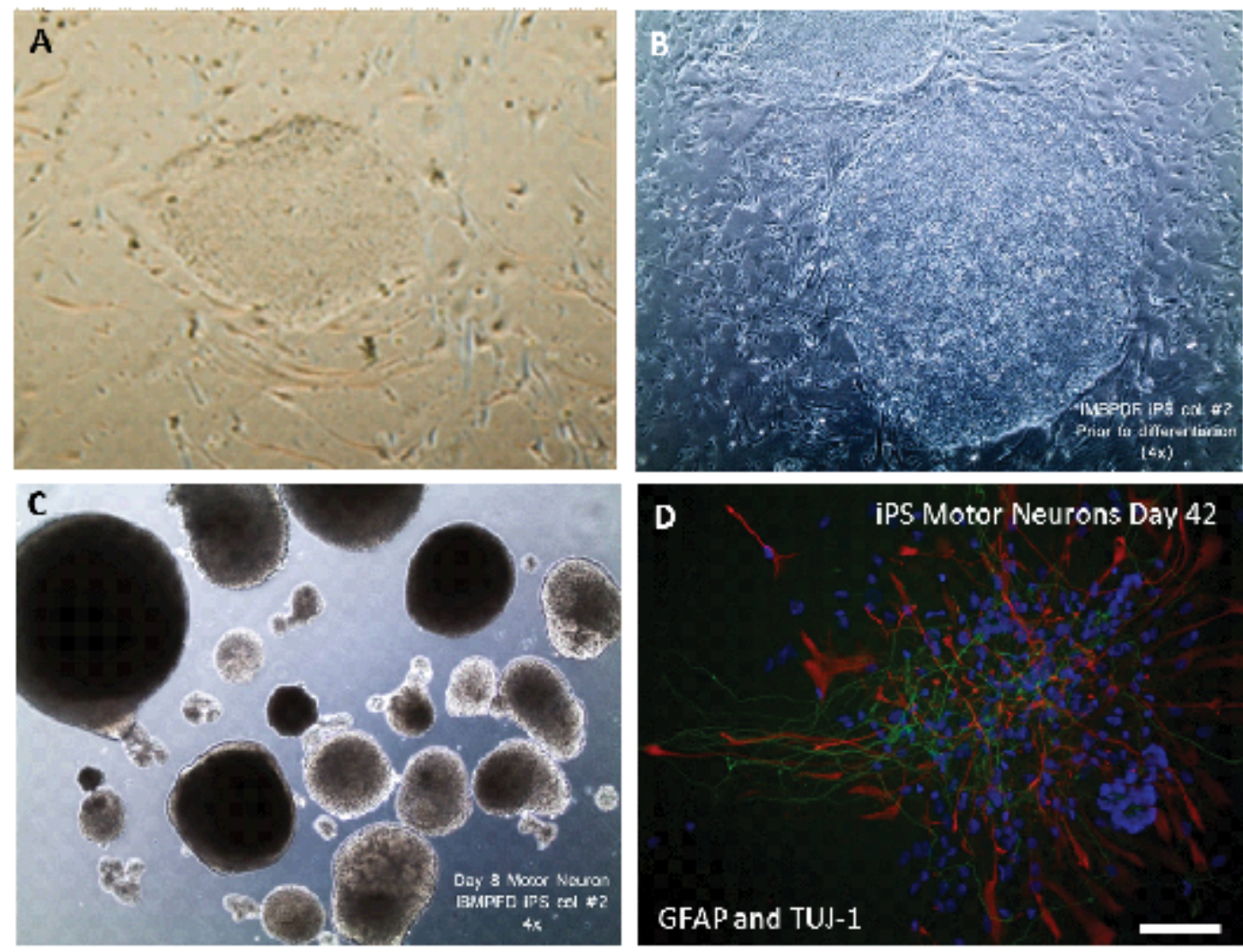

Figure 3: iPS colony formation and differentiation. Human VCP iPS colonies were analyzed by light microscopy. (A and B) Day 36 iPS undifferentiated colonies, (C) Day 8 iPS-derived motor neuron colony, and (D) Day 42 iPSC stained with GFAP (FITC) and TUJ-1 (TRITC). (Magnification 200X). 
Citation: Dec E, Ferguson D, Nalbandian A, Gargus M, Katheria V, et al. (2014) Disease-Specific Induced Pluripotent Stem Cell Modeling: Insights into the Pathophysiology of Valosin Containing Protein (VCP) Disease. J Stem Cell Res Ther 4: 168. doi:10.4172/2157-7633.1000168

Page 5 of 6
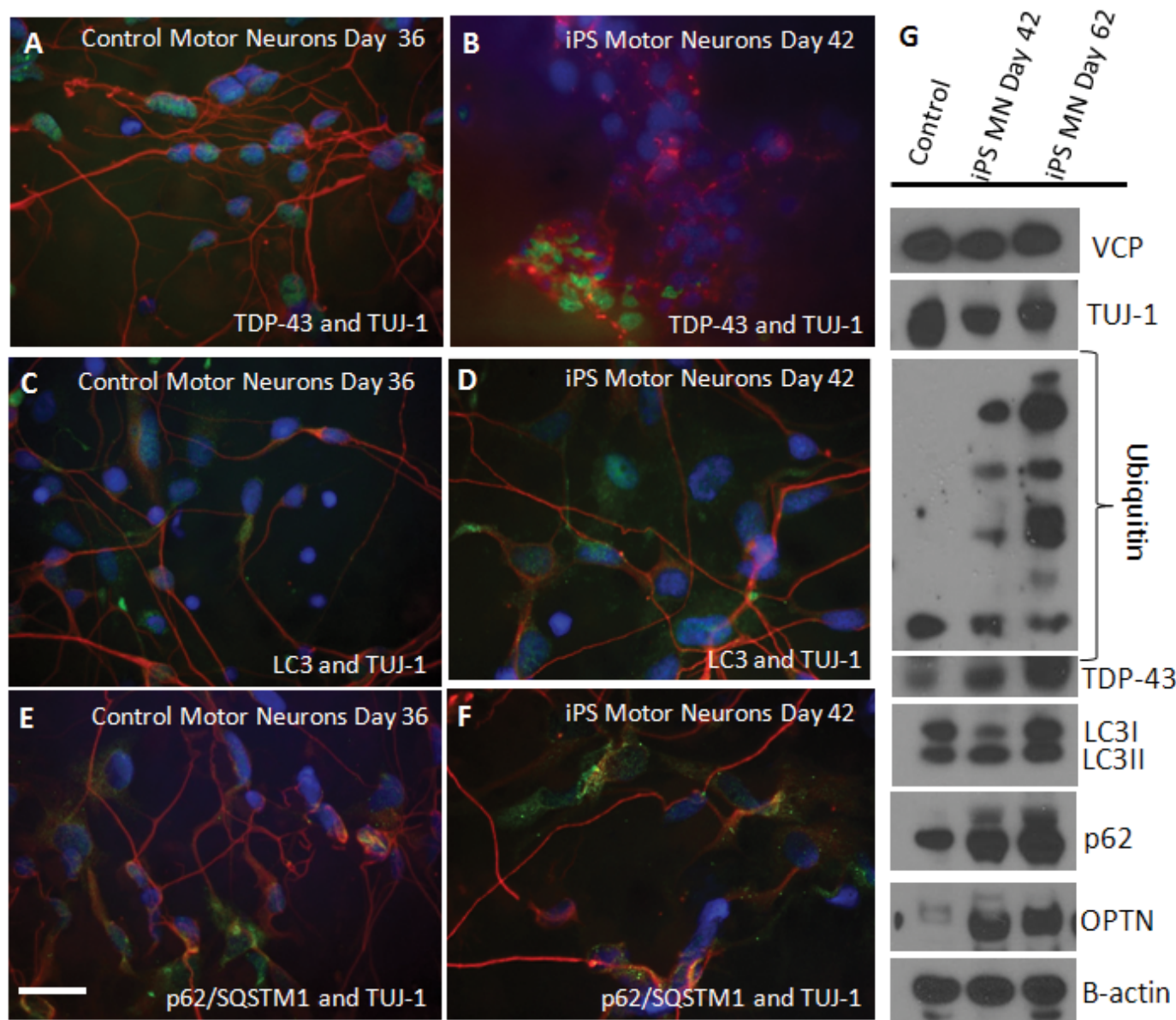

Figure 4: Differentiation of human iPSC into neurons. Control and differentiated iPSC-derived neural lineage were double immunostained with (A and B) TDP-43 (FITC) and TUJ-1 (TRITC), (C and D) LC3 (FITC) and TUJ-1 (TRITC), and (E and F) p62/SQSTM1 (FITC) and TUJ-1 (TRITC) (Magnification 200X). DAPI was used for nuclear staining. (G) Western blot analysis of markers: VCP, TUJ-1, Ubiquitin, TDP-43, LC3-I/II, p62/SQSTM1, and Optineurin (OPTN). 3 -actin was used as a loading control in these experiments.

tissue samples are difficult to obtain and normal primary cell line senescence hinders research progress. In this study, we describe a fully pluripotent iPSC model of VCP disease that was differentiated into a neuronal lineage, thereby providing a useful platform to explore the pathophysiological mechanisms.

Morphologically, we observed an elevation of the autophagy marker proteins TDP-43, LC3-I/II, p62/SQSTM1, optineurin, and ubiquitin in our human iPSC neural lineage, thereby suggesting an accumulation of these proteins at different subcellular levels in the autophagy signaling cascade, ultimately resulting in vacuolization and muscle atrophy. The homeostasis of skeletal muscles is dependent upon the proper regulation of autophagic flux and this has been shown to be disrupted in physiological and pathological conditions, recapitulating a hallmark of the VCP cellular phenotype.

Ultimately, understanding the cellular and molecular signaling transduction pathways affected by VCP mutations may hold future promise in the development, assessment, and clinical application of pharmacological and gene therapies for VCP-associated. The consistency, expandability, and purity of the hiPSC in VCP disease provides a very valuable tool and opportunity to screen and test drugs in vitro, thereby offering the significant possibility to intervene in the early stages of the disease. In this manner, the patient VCP iPSC model offers a unique platform in understanding the pathophysiology and new vistas in improving muscle integrity and/or slowing down the progression of muscle wasting in patients with VCP and other related neurodegenerative diseases.

\section{Acknowledgements}

We wish to thank Drs. Leslie Locke, Lesley Thompson, Peter Donovan, Lisa Flanagan, Edwin Monuki and Phillip Schwartz for their helpful discussions on the iPSC research. Funding for this study was provided by UC Irvine start-up funds to VEK and California Stem Cell Clinical Fellowship to EKD.

\section{Conflict of Interest}

The funders had no role in study design, data collection and analysis, decision to publish, or preparation of the manuscript. No conflicts of interest.

\section{References}

1. DeJesus-Hernandez M, Mackenzie IR, Boeve BF, Boxer AL, Baker M, et al (2011) Expanded GGGGCC hexanucleotide repeat in noncoding region of C9ORF72 causes chromosome 9p-linked FTD and ALS. Neuron 72: 245-256.

2. Johnson JO, Mandrioli J, Benatar M, Abramzon Y, Van Deerlin VM, et al (2010) Exome sequencing reveals VCP mutations as a cause of familial ALS Neuron 68: 857-864.

3. Schroder R, Watts GD, Mehta SG, Evert BO, Broich P, et al. (2005) Mutant valosin-containing protein causes a novel type of frontotemporal dementia. Ann Neurol 57: 457-461. 
Citation: Dec E, Ferguson D, Nalbandian A, Gargus M, Katheria V, et al. (2014) Disease-Specific Induced Pluripotent Stem Cell Modeling: Insights into the Pathophysiology of Valosin Containing Protein (VCP) Disease. J Stem Cell Res Ther 4: 168. doi:10.4172/2157-7633.1000168

Page 6 of 6

4. Djamshidian A, Schaefer J, Haubenberger D, Stogmann E, Zimprich F, et al. (2009) A novel mutation in the VCP gene (G157R) in a German family with inclusion-body myopathy with Paget disease of bone and frontotemporal dementia. Muscle Nerve 39: 389-391.

5. Guyant-Marechal L, Laquerriere A, Duyckaerts C, Dumanchin C, Bou J, et al. (2006) Valosin-containing protein gene mutations: clinical and neuropathologic features. Neurology 67: 644-651.

6. Haubenberger D, Bittner RE, Rauch-Shorny S, Zimprich F, Mannhalter C, et al. (2005) Inclusion body myopathy and Paget disease is linked to a novel mutation in the VCP gene. Neurology 65: 1304-1305.

7. Bersano A, Del Bo R, Lamperti C, Ghezzi S, Fagiolari G, et al. (2007) Inclusion body myopathy and frontotemporal dementia caused by a novel VCP mutation. Neurobiol Aging30: 752-758.

8. Viassolo V, Previtali SC, Schiatti E, Magnani G, Minetti C, et al. (2008) Inclusion body myopathy, Paget's disease of the bone and frontotemporal dementia: recurrence of the VCP R155H mutation in an Italian family and implications for genetic counselling. Clin Genet 74: 54-60.

9. Miller TD, Jackson AP, Barresi R, Smart CM, Eugenicos M, et al. (2009) Inclusion body myopathy with Paget disease and frontotemporal dementia (IBMPFD): clinical features including sphincter disturbance in a large pedigree. J Neurol Neurosurg Psychiatry 80: 583-584.

10. Kumar KR, Needham M, Mina K, Davis M, Brewer J, et al. (2010) Two Australian families with inclusion-body myopathy, Paget's disease of bone and frontotemporal dementia: novel clinical and genetic findings. Neuromuscul Disord 20: 330-334.

11. Fanganiello RD, Kimonis V, Nitrini R, Passos-Bueno MR (2011) A Brazilian family with IBMPFD caused by p.R93C mutation in the VCP gene and literature review for genotype-phenotype correlations. Experimental Brain Research.

12. Kim EJ, Park YE, Kim DS, Ahn BY, Kim HS, et al. (2011) Inclusion body myopathy with Paget disease of bone and frontotemporal dementia linked to VCP p.Arg155Cys in a Korean family. Arch Neurol 68: 787-796.

13. Komatsu J, Iwasa K, Yanase D, Yamada M (2013) Inclusion body myopathy with Paget disease of the bone and frontotemporal dementia associated with a novel G156S mutation in the VCP gene. Muscle Nerve.

14. Spina S, Van Laar A, Murrell JR, de Courten-Myers G, Hamilton RL, et al. (2008) Frontotemporal dementia associated with a Valosin-Containing Protein mutation: report of three families. The FASEB Journal 22: 58.54 .

15. Watts GD, Thomasova D, Ramdeen SK, Fulchiero EC, Mehta SG, et al. (2007) Novel VCP mutations in inclusion body myopathy associated with Paget disease of bone and frontotemporal dementia. Clin Genet 72: 420-426.

16. Chan N, Le C, Shieh P, Mozaffar P, Bronstein J, et al. (2010) Valosin-Containing Protein Mutation and Parkinson's Disease (in review) Neurology.

17. Shamirian S, Nalbandian A, Khare M, Castellani R, Kim R, et al. (2013) Earlyonset Alzheimers and Cortical Vision Impairment in a Woman With Valosincontaining Protein Disease Associated With 2 APOE [Latin Small Letter Open E]4/APOE [Latin Small Letter Open E]4 Genotype. Alzheimer Dis Assoc Disord.

18. Kimonis VE, Kovach MJ, Waggoner B, Leal S, Salam A, et al. (2000) Clinical and molecular studies in a unique family with autosomal dominant limb-girdle muscular dystrophy and Paget disease of bone. Genet Med 2: 232-241.

19. Meyer H, Bug M, Bremer S (2012) Emerging functions of the VCP/p97 AAAATPase in the ubiquitin system. Nat Cell Biol 14: 117-123.

20. Ju JS, Fuentealba RA, Miller SE, Jackson E, Piwnica-Worms D, et al. (2009) Valosin-containing protein (VCP) is required for autophagy and is disrupted in VCP disease. J Cell Biol 187: 875-888.

21. Vesa J, Su H, Watts GD, Krause S, Walter MC, et al. (2009) Valosin containing protein associated inclusion body myopathy: abnormal vacuolization, autophagy and cell fusion in myoblasts. Neuromuscul Disord 19: 766-772.

22. Kim NC, Tresse E, Kolaitis RM, Molliex A, Thomas RE, et al. (2013) VCP Is Essential for Mitochondrial Quality Control by PINK1/Parkin and this Function Is Impaired by VCP Mutations. Neuron 78: 65-80.

23. Nalbandian A, Ghimbovschi S, Radom-Aizik S, Dec E, Vesa J, et al. (2012) Global Gene Profiling of VCP-associated Inclusion Body Myopathy. Clin Transl Sci 5: 226-234.

24. Watts GD, Wymer J, Kovach MJ, Mehta SG, Mumm S, et al. (2004) Inclusion body myopathy associated with Paget disease of bone and frontotemporal dementia is caused by mutant valosin-containing protein. Nat Genet 36: 377 381 .

25. Kimonis VE, Fulchiero E, Vesa J, Watts G (2008) VCP disease associated with myopathy, paget disease of bone and frontotemporal dementia: Review of a unique disorder. Biochim Biophys Acta 1782: 744-748.

26. Kimonis VE, Mehta SG, Fulchiero EC, Thomasova D, Pasquali M, et al. (2008) Clinical studies in familial VCP myopathy associated with Paget disease of bone and frontotemporal dementia. Am J Med Genet A 146: 745-757.

27. Hutton M, Lendon CL, Rizzu P, Baker M, Froelich S, et al. (1998) Association of missense and 5'-splice-site mutations in tau with the inherited dementia FTDP17. Nature 393: 702-705.

28. Spillantini MG, Murrell JR, Goedert M, Farlow MR, Klug A, et al. (1998) Mutation in the tau gene in familial multiple system tauopathy with presenile dementia. Proc Natl Acad Sci U S A 95: 7737-7741.

29. Wilhelmsen KC, Blake DM, Lynch T, Mabutas J, De Vera M, et al. (1996) Chromosome 12-linked autosomal dominant scapuloperoneal muscular dystrophy. Ann Neurol 39: 507-520.

30. Baker M, Mackenzie IR, Pickering-Brown SM, Gass J, Rademakers R, et al. (2006) Mutations in progranulin cause tau-negative frontotemporal dementia linked to chromosome 17. Nature 442: 916-919.

31. Rollinson S, Snowden JS, Neary D, Morrison KE, Mann DM, et al. (2007) TDP43 gene analysis in frontotemporal lobar degeneration. Neurosci Lett 419: 1-4.

32. Urwin H, Josephs KA, Rohrer JD, Mackenzie IR, Neumann M, et al. (2010) FUS pathology defines the majority of tau- and TDP-43-negative frontotemporal lobar degeneration. Acta Neuropathol 120: 33-41.

33. Ju JS, Weihl CC (2010) p97/VCP at the intersection of the autophagy and the ubiquitin proteasome system. Autophagy 6: 283-285

34. Ju JS, Weihl CC (2010) Inclusion body myopathy, Paget's disease of the bone and fronto-temporal dementia: a disorder of autophagy. Hum Mol Genet 19: R38-45.

35. Mizushima N, Klionsky DJ (2007) Protein turnover via autophagy: implications for metabolism. Annu Rev Nutr 27: 19-40.

36. Moscat J, Diaz-Meco MT (2009) p62 at the crossroads of autophagy, apoptosis and cancer. Cell 137: 1001-1004.

37. Wang IF, Guo BS, Liu YC, Wu CC, Yang CH, et al. (2012) Autophagy activators rescue and alleviate pathogenesis of a mouse model with proteinopathies of the TAR DNA-binding protein 43. Proc Natl Acad Sci U S A 109: 15024-15029.

38. Takahashi K, Okita K, Nakagawa M, Yamanaka S (2007) Induction of pluripotent stem cells from fibroblast cultures. Nat Protoc 2: 3081-3089.

39. Takahashi K, Tanabe K, Ohnuki M, Narita M, Ichisaka T, et al. (2007) Induction of pluripotent stem cells from adult human fibroblasts by defined factors. Cell 131: $861-872$

40. Badadani M, Nalbandian A, Watts GD, Vesa J, Kitazawa M, et al. (2010) VCP associated inclusion body myopathy and paget disease of bone knock-in mouse model exhibits tissue pathology typical of human disease. PLoS One 5.

41. Nalbandian A, Llewellyn KJ, Badadani M, Yin HZ, Nguyen C, et al. (2013) A progressive translational mouse model of human valosin-containing protein disease: the VCP(R155H/+) mouse. Muscle Nerve 47: 260-270.

42. Kimonis V, Donkervoort S, Watts G (2011) Inclusion Body Myopathy Associated with Paget Disease of Bone and/or Frontotemporal Dementia GeneGeneTests (www.genetests.org) and University of Washington, Seattle, USA

43. Kimonis VE, Watts GD (2005) Autosomal dominant inclusion body myopathy, Paget disease of bone, and frontotemporal dementia. Alzheimer Dis Assoc Disord 19: S44-47.

44. Benatar M, Wuu J, Fernandez C, Weihl CC, Katzen H, et al. (2013) Motor neuron involvement in multisystem proteinopathy: Implications for ALS. Neurology 80: 1874-1880. 